\title{
Intra-articular angiofibroma of soft tissue of the knee: A case report
}

\author{
YUUYA HASHINO $^{1}$, JUN NISHIO ${ }^{1}$, AKIRA MAEYAMA ${ }^{1}$, \\ MIKIKO AOKI ${ }^{2}$, KAZUKI NABESHIMA ${ }^{2}$ and TAKUAKI YAMAMOTO ${ }^{1}$
}

\author{
Departments of ${ }^{1}$ Orthopaedic Surgery and ${ }^{2}$ Pathology, Faculty of Medicine, Fukuoka University, Fukuoka 814-0180, Japan
}

Received November 29, 2016; Accepted April 19, 2017

DOI: $10.3892 / \mathrm{mco} .2017 .1298$

\begin{abstract}
Angiofibroma of soft tissue (AFST) is an extremely rare soft tissue neoplasm that typically presents as a slow-growing, painless mass in the extremities. The present study reports an unusual case of an intra-articular AFST occurring in the left knee of a 23 -year-old female. Physical examination revealed a $3-\mathrm{cm}$, relatively mobile, elastic-hard, non-tender mass. Magnetic resonance imaging detected an intra-articular soft tissue mass with iso-signal intensity relative to skeletal muscle on T1-weighted sequences and heterogeneous high signal intensity on T2-weighted sequences. Contrast-enhanced fat-suppressed T1-weighted sequences demonstrated strong peripheral enhancement of the mass. An arthroscopic excision of the mass was performed. Histologically, the tumor was composed of spindle- or oval-shaped cells in a fibromyxoid stroma with a prominent vascular pattern. Immunohistochemically, the tumor cells were diffusely positive for vimentin and CD163 and focally positive for CD68, desmin and estrogen receptor. Based on these findings, the tumor was diagnosed as an AFST. The patient had no evidence of local recurrence within 9 months of follow-up. To the best of our knowledge, this is the first case of intra-articular AFST managed successfully with arthroscopic excision.
\end{abstract}

\section{Introduction}

Angiofibroma of soft tissue (AFST) is a recently described benign soft tissue tumor with distinctive histological features and a unique cytogenetic abnormality (1). It mainly arises in the deep soft tissue of the lower extremities and often affects middle-aged adults with a female predominance (2). AFST typically presents as a slow-growing, painless, well-defined mass. The lesions range from 1.2 to $12 \mathrm{~cm}$ in their greatest

Correspondence to: Dr Jun Nishio, Department of Orthopaedic Surgery, Faculty of Medicine, Fukuoka University, 7-45-1 Nanakuma, Jonan-ku, Fukuoka 814-0180, Japan

E-mail: jnishio@cis.fukuoka-u.ac.jp

Key words: angiofibroma, intra-articular, knee, magnetic resonance imaging, AHRR, NCOA2 diameter, with a median size of $3.5 \mathrm{~cm}$ (1). The tumor consists of a vaguely lobular, variably cellular proliferation of uniform, bland spindle cells with an abundant, variably myxoid or collagenous extracellular matrix and numerous small, thin-walled, branching blood vessels. Due to its fibrovascular morphology, the differential diagnosis of AFST includes a range of benign and low-grade malignant soft tissue tumors. Simple excision is curative and local recurrence is rare (1). The current report describes a case of AFST arising in the intra-articular location of a young adult female, which was successfully treated arthroscopically. Written informed consent for this study was obtained from the patient.

\section{Case report}

A 23-year-old female was referred to Fukuoka University Hospital (Fukuoka, Japan) with a 1-month history of a palpable mass in the anteromedial aspect of the left knee. Physical examination revealed a relatively mobile, elastic-hard, non-tender mass, measuring approximately $3.0 \times 3.0 \mathrm{~cm}$. The range of motion of the left knee was normal. Laboratory analyses were within normal limits. The patient's medical history was non-contributory.

Plain radiographs suggested the presence of a joint effusion. Magnetic resonance imaging (MRI) showed an intra-articular soft tissue mass. Mild synovial thickening was also observed with a small amount of joint effusion. The mass exhibited iso-signal intensity relative to skeletal muscle on T1-weighted sequences (Fig. 1A) and heterogeneous high signal intensity on T2-weighted sequences (Fig. 1B). Contrast-enhanced fat-suppressed T1-weighted sequences demonstrated strong peripheral enhancement of the mass (Fig. 1C). There was no evidence of bone involvement. A benign soft tissue tumor such as hemangioma or synovial plica was suggested, and the lesion was arthroscopically excised (Fig. 2).

Microscopically, the tumor was composed of spindle- or oval-shaped cells in a fibromyxoid stroma with a prominent vascular pattern (Fig. 3A). Neither cellular atypia nor mitotic figures were observed. Immunohistochemically, the tumor cells were diffusely positive for vimentin and CD163 (Fig. 3B) and focally positive for CD68, desmin (Fig. 3C), and estrogen receptor (ER) (Fig. 3D). Staining for cytokeratin, epithelial membrane antigen (EMA), calponin, smooth muscle actin (SMA), CD34 and S-100 protein was negative. Based on these 

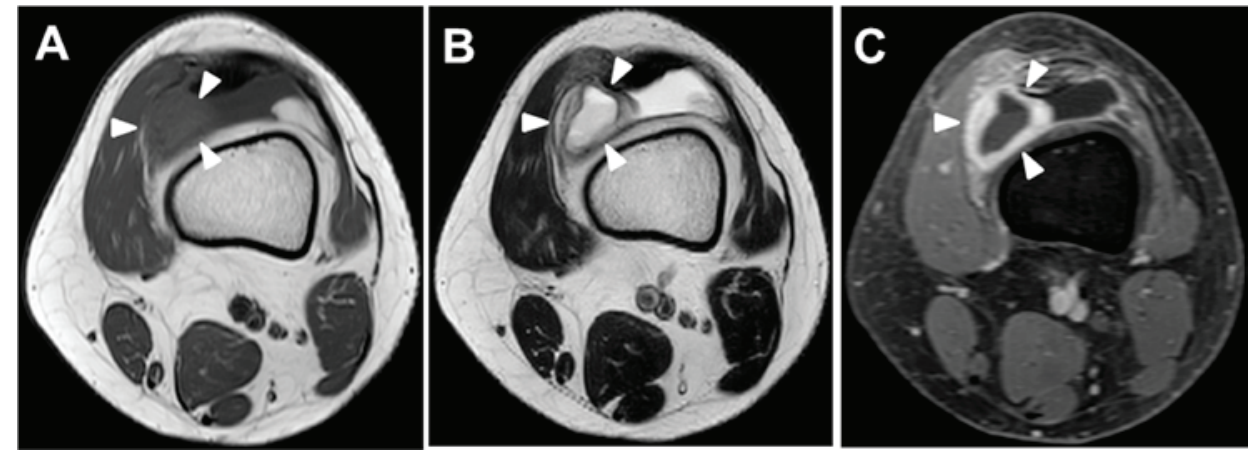

Figure 1. Axial magnetic resonance images of soft tissue angiofibroma in the left knee. (A) T1-weighted sequence shows that the mass (arrowheads) has iso-signal intensity relative to skeletal muscle. (B) T2-weighted sequence exhibits that the mass (arrowheads) has heterogeneous high signal intensity. (C) Contrast-enhanced fat-suppressed T1-weighted sequence demonstrates strong peripheral enhancement of the mass (arrowheads).

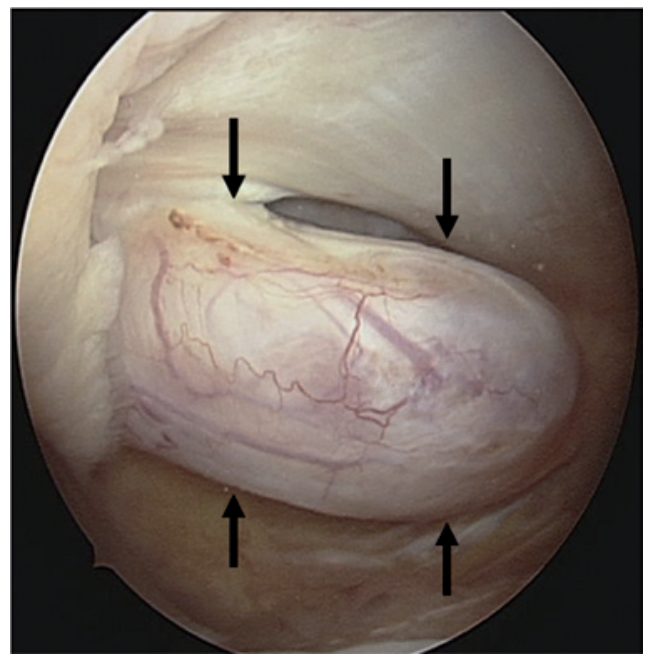

Figure 2. Arthroscopic view of the knee. The tumor (arrows) was present at the medial side of the suprapatellar pouch.
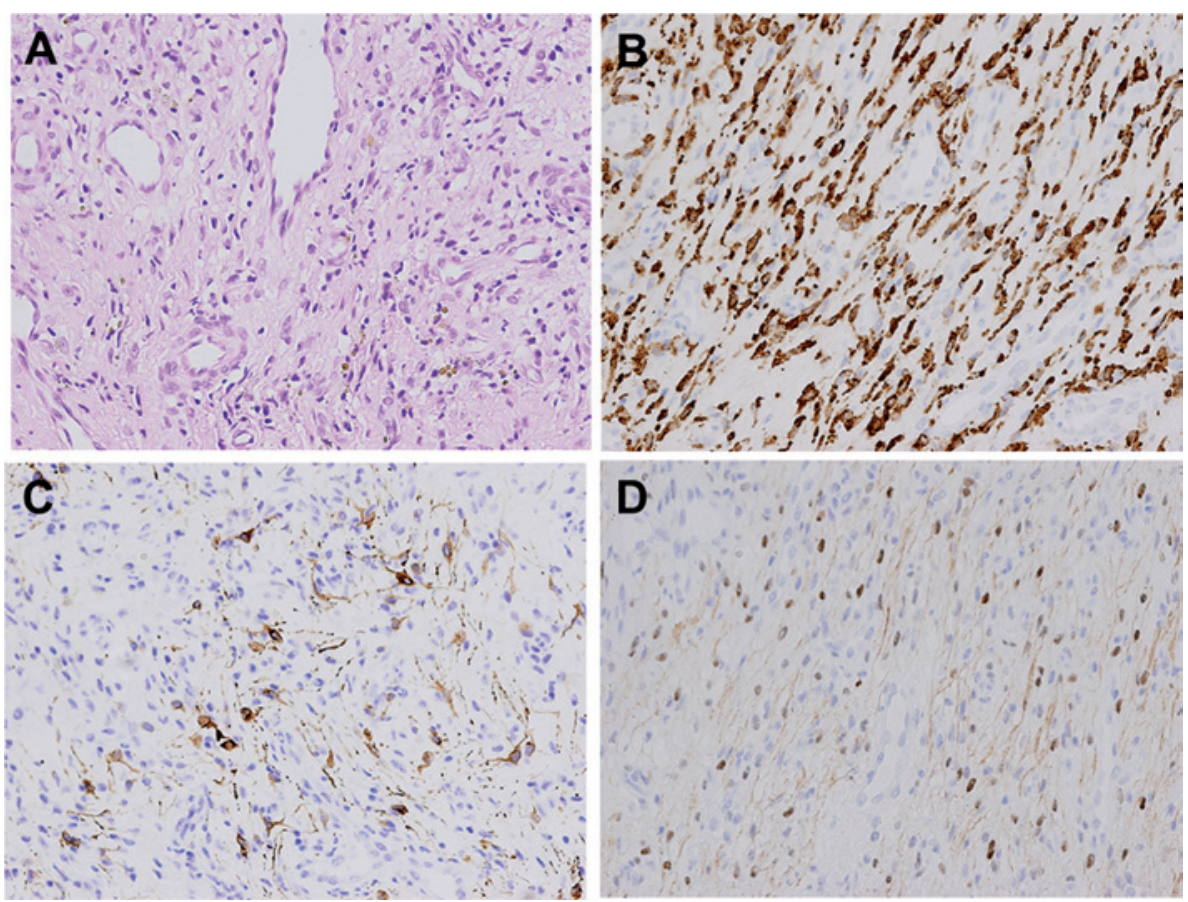

Figure 3. Histological and immunohistochemical findings of soft tissue angiofibroma. (A) The tumor is composed of spindle- or oval-shaped cells in a fibromyxoid stroma (magnification, x80). The tumor cells are (B) diffusely immunoreactive for CD163 and focally immunoreactive for (C) desmin and (D) estrogen receptor (magnification, $\mathrm{x} 80$ ). 
features, the tumor was diagnosed as an AFST. In the current case, an aryl hydrocarbon receptor repressor (AHRR)-nuclear receptor coactivator 2 (NCOA2) or NCOA2-AHRR fusion gene was not detected by reverse transcription polymerase chain reaction.

The postoperative course was uneventful. There was no clinical evidence of local recurrence at the 9-month follow-up.

\section{Discussion}

Angiofibroma of soft tissue (AFST) was first described by Mariño-Enríquez and Fletcher in 2012 (1). The exact etiology of AFST and its association with cellular angiofibroma remain unclear. Clinically, AFST behaves in a benign manner, and malignant transformation has not been reported (1). To the best of our knowledge, this is the first English-language case report of intra-articular AFST that provides detailed radiological and histological features.

Histologically, AFST is characterized by a proliferation of uniform, bland spindle tumor cells with abundant collagenous to myxoid stroma and prominent small, thin-walled, branching blood vessels (1). Mitotic activity is occasionally found, but cytologic atypia and nuclear hyperchromasia are absent. Immunohistochemically, expression of EMA was seen in $44 \%$ of the cases analyzed (1), whereas the current case did not exhibit any expression of EMA. Occasional cases may exhibit scattered cells that stain for CD34, SMA and desmin (2). Previous immunohistochemical studies have revealed that CD163 and ER are useful markers in the diagnosis of AFST $(3,4)$, as in the present case. S-100 protein expression is consistently negative (1).

Recent cytogenetic and molecular studies have identified a balanced $\mathrm{t}(5 ; 8)(\mathrm{p} 15 ; \mathrm{q} 13)$ translocation resulting in a fusion of AHRR and NCOA2 (5). In addition, Arbajian et al (6) demonstrated a general transcription factor IIi (GTF2I)-NCOA2 fusion gene in a AFST with a three-way $\mathrm{t}(7 ; 8 ; 14)(\mathrm{q} 11 ; \mathrm{q} 13 ; \mathrm{q} 31)$ translocation, emphasizing the role of $N C O A 2$. Furthermore, a small proportion of $N C O A 2$ gene rearrangement-positive cells have been observed in almost all cases by fluorescence in situ hybridization (3,7). However, it has been noted that a substantial subset of AFSTs were negative for the fusion gene $(3,5)$, as in the current case.

To the best of our knowledge, there is only one case report describing the radiological features of AFST (8). In that report, MRI revealed a circumscribed soft tissue mass with iso- to slightly-low signal intensity relative to skeletal muscle on T1-weighted sequences and markedly high signal intensity on fat-suppressed T2-weighted sequences. Contrast-enhanced fat-suppressed T1-weighted sequences revealed diffuse enhancement throughout the mass. On the other hand, MRI of the current case exhibited a well-defined mass with iso-signal intensity relative to skeletal muscle on T1-weighted sequences, heterogeneous high signal intensity on T2-weighted sequences, and strong peripheral enhancement on contrast-enhanced fat-suppressed T1-weighted sequences. Taken together, the MRI appearance of AFST may be variable and depends on the amount of spindle cells, myxoid and collagenous stroma and blood vessels within the tumor.

The histological differential diagnosis for the current lesion includes solitary fibrous tumor (SFT). In the current World
Health Organization classification, SFT is classified into the intermediate (rarely metastasizing) category (9). The spindle cell component in SFT has similar bland cytological features and patternless architecture to that in AFST (10). In addition, thin-walled branching hemangiopericytoma-like vessels are able to be found in both tumors. Compared with AFST, however, the tumor cells in SFT exhibit strong and diffuse expression of CD34 (9). Immunohistochemical studies have demonstrated that signal transducer and activator of transcription 6 (STAT6) is a highly sensitive and specific marker for the diagnosis of SFT $(11,12)$. Furthermore, a nerve growth factor inducible-A binding protein 2 (NAB2)-STAT6 fusion gene has been described as a novel molecular hallmark in SFT $(13,14)$.

In conclusion, the present study described the first case of an intra-articular AFST of the knee in a young adult female. It is important to be familiar with the clinicopathological and radiological features of this entity for its accurate diagnosis and appropriate treatment.

\section{Acknowledgements}

The authors would like to thank Dr. Yoshinao Oda (Department of Anatomic Pathology, Graduate School of Medicine, Kyushu University, Fukuoka, Japan) for his expert opinion and valuable comments on the histological diagnosis of this case.

\section{References}

1. Mariño-Enríquez A and Fletcher CDM: Angiofibroma of soft tissue: Clinicopathologic characterization of a distinctive benign fibrovascular neoplasm in a series of 37 cases. Am J Surg Pathol 36: 500-508, 2012.

2. Nishio J: Updates on the cytogenetics and molecular cytogenetics of benign and intermediate soft tissue tumors. Oncol Lett 5: 12-18, 2013.

3. Yamada Y, Yamamoto H, Kohashi K, Ishii T, Iura K, Maekawa A, Bekki H, Otsuka H, Yamashita K, Tanaka H, et al: Histological spectrum of angiofibroma of soft tissue: Histological and genetic analysis of 13 cases. Histopathology 69: 459-469, 2016.

4. Fukuda Y, Motoi T, Kato I, Ikegami M, Funata N, Ohtomo R, Horiguchi S, Goto T and Hishima T: Angiofibroma of soft tissue with fibrohistiocytic features and intratumor genetic heterogeneity of NCOA2 gene rearrangement revealed by chromogenic in situ hybridization: A case report. Pathol Int 64: 237-242, 2014.

5. Jin Y, Möller E, Nord KH, Mandahl N, Von Steyern FV, Domanski HA, Mariño-Enríquez A, Magnusson L, Nilsson J, Sciot R, et al: Fusion of the AHRR and NCOA2 genes through a recurrent translocation $\mathrm{t}(5 ; 8)(\mathrm{p} 15 ; \mathrm{q} 13)$ in soft tissue angiofibroma results in upregulation of aryl hydrocarbon receptor target genes. Genes Chromosomes Cancer 51: 510-520, 2012.

6. Arbajian E, Magnusson L, Mertens F, Domanski HA, Vult von Steyern F and Nord KH: A novel GTF2I/NCOA2 fusion gene emphasizes the role of NCOA2 in soft tissue angiofibroma development. Genes Chromosomes Cancer 52: 330-331, 2013.

7. Sugita S, Aoyama T, Kondo K, Keira Y, Ogino J, Nakanishi K, Kaya M, Emori M, Tsukahara T, Nakajima H, et al: Diagnostic utility of NCOA2 fluorescence in situ hybridization and Stat6 immunohistochemistry staining for soft tissue angiofibroma and morphologically similar fibrovascular tumors. Hum Pathol 45: 1588-1596, 2014

8. Lee JJ, Bredella MA, Springfield DS and Nielsen GP: Soft tissue angiofibroma: A case report. Skeletal Radiol 43: 403-407, 2014.

9. Fletcher CDM, Bridge JA and Lee JC: Extrapleural solitary fibrous tumor. In: World Health Organization Classification of Tumours of Soft Tissue and Bone. Fletcher CDM, Bridge JA, Hogendoorn PCW and Mertens F (eds). IARC Press, Lyon, pp80-82, 2013.

10. Edgar MA, Lauer SR, Bridge JA and Rizzo M: Soft tissue angiofibroma: Report of 2 cases of a recently described tumor. Hum Pathol 44: 438-441, 2013. 
11. Doyle LA, Vivero M, Fletcher CD, Mertens F and Hornick JL: Nuclear expression of STAT6 distinguishes solitary fibrous tumor from histologic mimics. Mod Pathol 27: 390-395, 2014.

12. Yoshida A, Tsuta K, Ohno M, Yoshida M, Narita Y, Kawai A, Asamura H and Kushima R: STAT6 immunohistochemistry is helpful in the diagnosis of solitary fibrous tumors. Am J Surg Pathol 38: 552-559, 2014.

13. Robinson DR, Wu YM, Kalyana-Sundaram S, Cao X, Lonigro RJ, Sung YS, Chen CL, Zhang L, Wang R, Su F, et al: Identification of recurrent NAB2-STAT6 gene fusions in solitary fibrous tumor by integrative sequencing. Nat Genet 45: 180-185, 2013.
14. Mohajeri A, Tayebwa J, Collin A, Nilsson J, Magnusson L, von Steyern FV, Brosjö O, Domanski HA, Larsson O, Sciot R, et al: Comprehensive genetic analysis identifies a pathognomonic NAB2/STAT6 fusion gene, nonrandom secondary genomic imbalances, and a characteristic gene expression profile in solitary fibrous tumor. Genes Chromosomes Cancer 52: 873-886, 2013 